\title{
Non-Stop Decay
}

National Cancer Institute

\section{Source}

National Cancer Institute. Non-Stop Decay. NCI Thesaurus. Code C29647.

In eukaryotes, the non-stop decay mechanism recognizes and degrades mRNAs that lack a stop codon. It releases ribosomes stalled at the 3' end of a mRNA and stimulates the exosome to rapidly degrade the transcript. (from PubMed 12210514) 